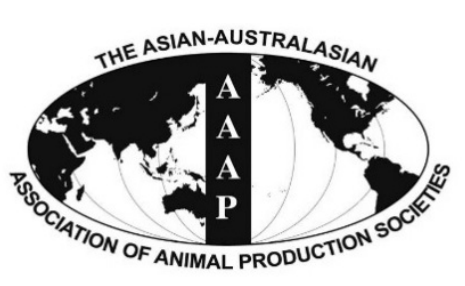

Open Access

Asian Australas. J. Anim. Sci.

Vol. 28, No. 10 : 1465-1470 October 2015

http://dx.doi.org/10.5713/ajas.14.0604

www.ajas.info

pISSN 1011-2367 elSSN 1976-5517

\title{
Effects of Dietary L-carnosine and Alpha-lipoic Acid on Growth Performance, Blood Thyroid Hormones and Lipid Profiles in Finishing Pigs
}

\author{
Yinghui Bao ${ }^{1, a}$, Chunqi Gao ${ }^{1,2, a}$, Wenbo Hao ${ }^{1}$, Cheng $\mathrm{Ji}^{1}$, Lihong $\mathrm{Zhao}^{1}$, \\ Jianyun Zhang ${ }^{1}$, Tao Liu ${ }^{1}$, and Qiugang Ma ${ }^{1, *}$ \\ ${ }^{1}$ State Key Laboratory of Animal Nutrition, College of Animal Science and Technology, \\ China Agricultural University, Beijing 100193, China
}

\begin{abstract}
The present study was conducted to determine the effects of L-carnosine (LC) and/or alpha-lipoic acid (ALA) supplementation on growth performance, blood thyroid hormones and lipid profiles in finishing pigs. A total of 40 (Landrace $\times$ Yorkshire) pigs with an initial body weight of $57.93 \pm 3.14 \mathrm{~kg}$ were randomly allocated to 4 experimental diets using a $2 \times 2$ factorial arrangement with 2 LC supplemental levels ( 0 or $0.1 \%$ ) and 2 ALA supplemental levels ( 0 or $0.03 \%)$ in basal diets. The results showed that pigs fed LC-supplemented diets increased final live weight, average daily gain, and average daily feed intake compared to those of pigs fed without LC-supplemented diets $(\mathrm{p}<0.05)$. Dietary supplementation with ALA did not affect the growth performance and carcass traits of pigs $(p>0.05)$. Additionally, LC supplementation increased serum triiodothyronine, thyroxine levels, and ALA supplementation increased serum triiodothyronine levels $(\mathrm{p}<0.05)$. Serum total cholesterol and triglycerides levels were significantly decreased in LC and ALA supplemented groups, respectively $(\mathrm{p}<0.05)$. Moreover, serum low density lipoprotein cholesterol levels were lower in the ALAsupplemented groups than those of pigs fed without ALA-supplemented diets $(p<0.05)$. However, no significant LC $\times$ ALA interaction effect on growth performance, blood thyroid hormones and lipid profiles was found. This study suggested that dietary supplementation of LC resulted in better growth performance compared to that of ALA supplementation. L-carnosine and/or ALA supplementation positively modified blood lipid profiles, which may have the potential to prevent cardiovascular diseases. (Key Words: Alpha-lipoic Acid, L-carnosine, Thyroid Hormones, Lipid Profiles, Finishing Pigs)
\end{abstract}

\section{INTRODUCTION}

L-carnosine ( $\beta$-alanyl-L-histidine, LC) is a dipeptide made up of $\beta$-alanine and L-histidine, which is highly concentrated in muscle and brain tissues of animals. Lcarnosine performs multiple biological functions including $\mathrm{pH}$ buffering, anti-oxidation, anti-glycation, anti-aging, and chelation of divalent metal cations (Babizhayev, 2006; Liu et al., 2008; Bellia et al., 2012). Ma et al. (2010) conducted a study to evaluate the effect of dietary $0.01 \%$ LC on improving pork quality by reducing the oxidative damages

\footnotetext{
* Corresponding Author: Qiugang Ma. Tel: +86-10-62732774, Fax: +86-10-62732774, E-mail: maqiugang@cau.edu.cn

${ }^{2}$ College of Animal Science, South China Agricultural University, Guangzhou 510642, China.

${ }^{\text {a }}$ These authors contributed equally to this work.

Submitted Aug. 8, 2014; Revised Oct. 2, 2014; Accepted Apr. 11, 2015
}

and enhancing meat stability in storage by its antioxidant activity. Furthermore, LC treatments ( 1 to $2 \mathrm{~g} / \mathrm{L}$ in drinking water) significantly decreased the mRNA expression of malic enzyme, fatty acid synthase and 3-hydroxy-3methylglutaryl coenzyme in liver, which subsequently declined triglycerides (TG) levels in plasma of mice that consumed high saturated fat diets (Mong et al., 2011; Brown et al., 2014). Similarly, a previous study demonstrated that supplementing rats diet with $0.5 \%$ LC decreases serum TG and low density lipoprotein cholesterol (LDL-C) levels (Kim et al., 2011). However, little is known about the effects of dietary LC supplementation on growth performance and lipid metabolism of pigs.

Alpha-lipoic acid (ALA) is a vitamin-like substance synthesized from octanoic acid in the mitochondrion (Bustamante et al., 1998; Roy and Packer, 1998) and a powerful micronutrient with various biological functions 
(Kim et al., 2011). Previous studies have found that the supplementation of $0.03 \%$ ALA into diets could enhance the serum levels of superoxide dismutase (SOD) and glutathione peroxidase (GSH-Px) in broilers (Zhang et al., 2009; Chen et al., 2011) and pigs (Bai et al., 2012). Moreover, ALA is an important cofactor in biological systems and serves a critical role in mitochondrial energy metabolism and lipid metabolism (Hamano, 2007; Shay et al., 2009). These metabolic effects of ALA may also improve growth performance and lipid metabolism of pigs. However, several publications have reported conflicting findings. For example, one reported negative growth performance in ALA-fed broilers (EI-Senousey et al., 2013), while another study reported ALA had no effect on growth performance in broilers (Hamano et al., 1999). Moreover, Kim et al. (2011) demonstrated that $0.5 \%$ ALA administration decreases serum TG significantly in rats. Similarly, previous studies demonstrated that supplementing broilers diet with $0.5 \%$ ALA decreases serum TG and total cholesterol (TC) levels (Hamano et al., 1999;2000). In contrast, an increased level of TG in serum was observed when broilers were fed with $0.02 \%$ ALA-supplemented diet (Hamano, 2006). Thus, whether the use of ALA improves growth performance and lipid metabolism of pigs is still unclear.

Kim et al. (2011) demonstrated that the combined effects of LC and ALA improve lipid metabolism in mice fed with high fat diet. However, the synergistic or additive effects of LC and ALA in pigs have not been investigated so far. Therefore, the objective of this study was to assess whether dietary LC and ALA, both alone and in combination can exact a positive effect on the growth performance, and the positive response was interrelated to blood thyroid hormones changes and lipid profiles in pigs.

\section{MATERIALS AND METHODS}

\section{Animals and experimental diets}

This study was approved by the Animal Care and Use Committee of China Agricultural University.

A total of 40 (Landrace $\times$ Yorkshire) pigs were randomly allocated to 4 experimental diets using a $2 \times 2$ factorial arrangement with 2 LC supplemental levels ( 0 or $0.1 \%$ ) and 2 ALA supplemental levels ( 0 or $0.03 \%$ ) in basal diets. Pigs were assigned by sex and weight in a randomized complete block design. Each treatment consisted of ten replicates (pens) with 1 pig each and the initial body weights (BW) of pigs were $57.93 \pm 3.14 \mathrm{~kg}$. The basal diet (Table 1) was based on corn-soybean meal to meet or exceed nutrient requirements for pigs of National Research Council (1998).

\section{Feeding and slaughter procedure}

The feeding research was carried out in Wenlai Pig
Table 1. The ingredients and nutrient concentrations of the control diet (as-fed basis)

\begin{tabular}{lc}
\hline Item $(\%$ unless note) & Content \\
\hline Ingredients & 73.23 \\
Corn & 18.07 \\
Soybean meal & 5.00 \\
Wheat bran & 1.06 \\
Limestone & 0.80 \\
Dicalcium phosphate & 0.44 \\
Salt & 0.40 \\
Soybean oil & 1.00 \\
Vitamin and trace mineral premix $^{1}$ & 100.00 \\
Total $_{\text {Nutrient content }}$ & \\
Metabolizable energy $^{2}(\mathrm{MJ} / \mathrm{kg})$ & 13.17 \\
Crude protein & \\
Calcium $^{3}$ & 15.50 \\
Total phosphorus $^{3}$ & 0.70 \\
Available phosphorus $^{2}$ & 0.60 \\
Lysine $^{3}$ & 0.40 \\
Methionine $^{3}$ & 0.90 \\
Methionine+crystine $^{2}$ & 0.28 \\
Tryptophane $^{2}$ & 0.54 \\
\hline
\end{tabular}

${ }^{1}$ Providing the following: $1,750 \mathrm{IU} / \mathrm{kg}$ vitamin $\mathrm{A}, 220 \mathrm{IU} / \mathrm{kg}$ vitamin $\mathrm{D}_{3}$, $23 \mathrm{IU} / \mathrm{kg}$ vitamin $\mathrm{E}, 0.55 \mathrm{mg} / \mathrm{kg}$ vitamin $\mathrm{K}_{3}, 0.25 \mathrm{mg} / \mathrm{kg}$ vitamin $\mathrm{B}_{1}, 1.0$ $\mathrm{mg} / \mathrm{kg}$ vitamin $B_{2}, 0.7 \mathrm{mg} / \mathrm{kg}$ vitamin $B_{6}, 31 \mathrm{~g} / \mathrm{kg}$ vitamin $B_{12}, 4 \mathrm{mg} / \mathrm{kg}$ niacin, $1.6 \mathrm{mg} / \mathrm{kg}$ calcium pantothenate, $0.1 \mathrm{mg} / \mathrm{kg}$ folic acid, $71 \mathrm{~g} / \mathrm{kg}$ biotic, $0.08 \mathrm{~g} / \mathrm{kg}$ choline chloride, $6.5 \mathrm{mg} / \mathrm{kg}$ manganese (as manganese sulfate), $15 \mathrm{mg} / \mathrm{kg}$ iron (as iron sulfate), $15 \mathrm{mg} / \mathrm{kg}$ zinc (as zinc oxide), $1.5 \mathrm{mg} / \mathrm{kg}$ copper (as copper sulfate), $0.07 \mathrm{mg} / \mathrm{kg}$ iodine (as potassium iodate), $0.03 \mathrm{mg} / \mathrm{kg}$ selenium (as sodium selenite), and $1 \mathrm{~g} / \mathrm{kg}$ sodium chloride.

${ }^{2}$ Calculated values.

${ }^{3}$ Determined values.

Farm (Shandong, China). During the experimental period, pigs were allowed ad libitum access to experimental diets and water. The amount of feed consumed was recorded daily for calculation of feed intake and the feed to gain ratio. Body weights were measured at the beginning and the termination of the experiment using a truck scales after feed was withheld for 16 hours.

At the end of the experiment, 16 hours after last feeding, all pigs were slaughtered. The slaughter plant used electrical stunning and a traditional scalding-singeing process. Following electrical stunning, after the pigs were exsanguinated, backfat thickness, and loin-eye area at the tenth rib were measured immediately postmortem according to the Chinese Guidelines on Performance Measurement Technology and Regulations for Pigs. Backfat thickness was the average of measurements at three points: the first rib, the last rib, and the last lumbar vertebra.

\section{Sample collection and serum biochemical parameters analysis}

Prior to slaughter, blood samples were collected from 
the anterior vena cava of the pigs. Ten milliliters of blood was harvested and centrifuged at 3,000 $\mathrm{g}$ for $10 \mathrm{~min}$ at $4^{\circ} \mathrm{C}$. Serum was stored at $-20^{\circ} \mathrm{C}$ for subsequent blood parameter analysis.

Serum growth hormone $(\mathrm{GH})$, triiodothyronine $\left(\mathrm{T}_{3}\right)$, thyroxine $\left(\mathrm{T}_{4}\right)$, and insulin-like growth factor (IGF)-1 concentrations were determined using assay kits purchased from Nanjing Jiancheng Bioengineering Institute with radioimmunoassay counter (Model xh6020, 262 national Nuclear Co., Beijing, China). Serum urea nitrogen (BUN), glucose, TC, TG, high density lipoprotein cholesterol (HDL-C), LDL-C, and very low density lipoprotein cholesterol (VLDL-C) levels were determined with the commercial diagnostic kits (Nanjing Jiancheng Bioengineering Institute, Nanjing, China) and the automatic biochemical analyzer (CE-CX5, Beckman Corp., Fullerton, CA, USA).

\section{Statistical analysis}

All the data were subjected to a one-way analysis of variance procedure, and the means were separated using Fisher's least significant difference multiple range test (SAS Institute, 2001). The four treatment groups were analyzed as a $2 \times 2$ factorial arrangement using the general linear model procedure in the SAS software (SAS Institute Inc., Cary, NC, USA). The model included the main effects of the LC and ALA as well as the LC $\times$ ALA interaction. The results were presented by mean values and the standard error of the mean. Effects were considered significant when $p<0.05$.

\section{RESULTS}

\section{Growth performance and carcass traits}

Results of the dietary treatments on pig growth performance and carcass traits are presented in Table 2. Compared with pigs fed without LC-supplemented diets, dietary supplementation of LC significantly increased the final live weight, average daily feed intake, and average daily gain (ADG) of pigs at the BW of 60 to $110 \mathrm{~kg}$ $(p<0.05)$. However, a diet supplemented with ALA did not affect the expression of growth performance and meat quality ( $p>0.05)$. No significant LC $\times$ ALA interaction effect on growth performance and carcass traits of pigs was found in the study $(\mathrm{p}>0.05)$.

\section{Measurements}

Serum hormones levels were affected by dietary ALA or LC (Table 3). Pigs fed $0.1 \%$ LC-supplemented diets had significantly increased serum $\mathrm{T}_{3}$ levels and $\mathrm{T}_{4}$ levels compared to those of pigs fed without LC-supplemented diets $(p<0.05)$. Moreover, the serum $\mathrm{T}_{3}$ levels of pigs were increased in $0.03 \%$ ALA-supplemented groups than those of pigs fed without ALA-supplemented diets $(p<0.05)$. However, synergistic effects by combined LC and ALA supplementation were not indicated by serum hormones levels $(p>0.05)$. There were no significant differences in serum IGF-1, GH, glucose, and BUN levels among treatments during the experiment $(\mathrm{p}>0.05)$.

The lipid profiles in the serum of pigs are presented in Table 4. Pigs fed with $0.1 \%$ LC-supplemented diets significantly reduced serum TC levels compared to those of pigs fed without LC-supplemented diets. Moreover, pigs fed $0.03 \%$ ALA-supplemented diet significantly reduced serum TG levels compared to those of pigs fed without ALAsupplemented diets $(\mathrm{p}<0.05)$. No significantly difference in serum HDL-C and VLDL-C levels were observed among the groups $(\mathrm{p}>0.05)$, whereas serum LDL-C levels were significantly lower in the ALA-supplemented groups than that of other group $(\mathrm{p}<0.05)$

\section{DISCUSSION}

L-carnosine and ALA are powerful micronutrients with various biological functions and are often used as health

Table 2. Effect of dietary L-carnosine (LC) and alpha-lipoid acid (ALA) supplementation on growth performance and carcass traits of finishing pigs ${ }^{1}$

\begin{tabular}{|c|c|c|c|c|c|c|c|c|}
\hline & \multicolumn{4}{|c|}{ Treatment $^{2}$} & \multirow{3}{*}{ SEM } & \multirow{2}{*}{\multicolumn{3}{|c|}{ Significance (p-value) }} \\
\hline & \multicolumn{2}{|c|}{ LC 0} & \multicolumn{2}{|c|}{ LC $0.1 \%$} & & & & \\
\hline & ALA 0 & ALA $0.03 \%$ & ALA 0 & ALA $0.03 \%$ & & $\mathrm{LC}$ & ALA & $\mathrm{LC}^{2} \mathrm{ALA}^{3}$ \\
\hline Final live weight (kg) & 97.44 & 99.28 & 103.12 & 103.20 & 0.89 & $<0.01$ & 0.628 & 0.604 \\
\hline $\operatorname{ADG}(\mathrm{g} / \mathrm{d})$ & 778.11 & 809.06 & 858.87 & 849.06 & 10.67 & $<0.01$ & 0.667 & 0.324 \\
\hline ADFI (g/d) & $2,485.32$ & $2,557.77$ & $2,793.74$ & $2,702.08$ & 50.60 & $<0.05$ & 0.927 & 0.388 \\
\hline Gain:feed (g/g) & 0.31 & 0.32 & 0.31 & 0.32 & 0.04 & 0.587 & 0.567 & 0.770 \\
\hline Carcass weight (kg) & 67.47 & 69.60 & 68.27 & 67.49 & 0.58 & 0.576 & 0.564 & 0.219 \\
\hline Carcass yield (\%) & 67.37 & 67.39 & 67.06 & 67.59 & 0.01 & 0.907 & 0.726 & 0.551 \\
\hline Longissimus muscle area $\left(\mathrm{cm}^{2}\right)$ & 33.48 & 33.47 & 34.24 & 34.56 & 0.65 & 0.533 & 0.766 & 0.468 \\
\hline Backfat (mm) & 14.20 & 14.33 & 15.00 & 13.40 & 0.42 & 0.691 & $<0.01$ & 0.797 \\
\hline
\end{tabular}

SEM, pooled standard error of the means; ADG, average daily gain; ADFI, average daily feed intake.

${ }^{1}$ Data were means of 10 pigs from per treatment.

${ }^{2}$ A $2 \times 2$ factorial arrangement with 2 supplemental levels of LC ( 0 or $\left.0.1 \%\right)$ and 2 supplemental levels of ALA ( 0 or $\left.0.03 \%\right)$ in basal diets.

${ }^{3} \mathrm{LC} \times \mathrm{ALA}$, the interaction between LC and ALA. 
Table 3. Effect of dietary L-carnosine (LC) and alpha-lipoid acid (ALA) supplementation on serum biochemical parameters levels in finishing pigs ${ }^{1}$

\begin{tabular}{|c|c|c|c|c|c|c|c|c|}
\hline & \multicolumn{4}{|c|}{ Treatment $^{2}$} & \multirow{3}{*}{ SEM } & \multirow{2}{*}{\multicolumn{3}{|c|}{ Significance (p-value) }} \\
\hline & \multicolumn{2}{|c|}{ LC 0} & \multicolumn{2}{|c|}{ LC $0.1 \%$} & & & & \\
\hline & ALA 0 & ALA $0.03 \%$ & ALA 0 & ALA $0.03 \%$ & & $\mathrm{LC}$ & ALA & $\mathrm{LC} \times \mathrm{ALA}^{3}$ \\
\hline $\mathrm{T}_{3}(\mathrm{ng} / \mathrm{mL})$ & 0.60 & 0.79 & 0.78 & 1.05 & 0.05 & $<0.01$ & $<0.01$ & 0.568 \\
\hline $\mathrm{T}_{4}(\mathrm{ng} / \mathrm{mL})$ & 35.37 & 41.06 & 43.33 & 44.64 & 1.23 & $<0.05$ & 0.101 & 0.294 \\
\hline $\mathrm{T}_{3} / \mathrm{T}_{4}$ & 1.74 & 1.94 & 1.83 & 2.36 & 0.10 & 0.202 & 0.077 & 0.398 \\
\hline IGF-1 (ng/mL) & 170.30 & 179.71 & 203.84 & 175.22 & 5.08 & 0.686 & 0.163 & 0.458 \\
\hline $\mathrm{GH}(\mathrm{ng} / \mathrm{mL})$ & 2.83 & 3.13 & 2.39 & 2.79 & 0.14 & 0.185 & 0.232 & 0.868 \\
\hline Glucose (mmol/L) & 4.55 & 4.51 & 5.31 & 5.22 & 0.19 & 0.069 & 0.871 & 0.945 \\
\hline $\mathrm{BUN}(\mathrm{mmol} / \mathrm{L})$ & 6.74 & 7.22 & 6.30 & 6.51 & 0.19 & 0.519 & 0.247 & 0.849 \\
\hline
\end{tabular}

SEM, pooled standard error of the means; $\mathrm{T}_{3}$, triiodothyronine; $\mathrm{T}_{4}$, thyroxine; IGF-1, insulin-like growth factor-1; GH, growth hormone; BUN, urea nitrogen.

${ }^{1}$ Data were means of 10 pigs from per treatment.

${ }^{2}$ A $2 \times 2$ factorial arrangement with 2 supplemental levels of LC ( 0 or $\left.0.1 \%\right)$ and 2 supplemental levels of ALA ( 0 or $\left.0.03 \%\right)$ in basal diets.

${ }^{3} \mathrm{LC} \times \mathrm{ALA}$, the interaction between LC and ALA.

supplements (Kim et al., 2011). Previous studies from our laboratory or other groups have shown that addition of LC or ALA into diets could enhance antioxidant capability and improve lipid metabolism in rats and broilers (Zhang et al., 2009; Chen et al., 2011; Kim et al., 2011). Thus, the supplementation of LC and ALA in diet may improve growth performance of pigs, although evidence remains conflicting (Hamano et al., 1999; Chen et al., 2011).

In the present study, the growth performance results indicated that, compared with pigs fed without LCsupplemented diets, supplementing LC to swine diets could improve the ADG of pigs at the weight of 60 to $110 \mathrm{~kg}$. Previous study reported that the thyroid hormones could stimulate nuclear accumulation and proliferation within skeletal muscle fibers, and influence the growth of skeletal muscles in animals (Smith et al., 2002). Thus, the growthpromoting effect of LC or LC+ALA may be partly related to thyroid hormone changes in pigs. To explore the relationship of animal growth and serum hormones, the serum thyroid hormones, including $\mathrm{T}_{3}$ and $\mathrm{T}_{4}$ were determined in the present study. The results showed that there was a significant increase in serum $T_{3}$ and $T_{4}$ in pigs fed with $0.1 \%$ LC. Higher serum $\mathrm{T}_{3}$ and $\mathrm{T}_{4}$ levels may induce proliferation of satellite cells and increase myofiber protein accretion (Qiao et al., 2013), which was in agreement with the enhanced ADG. Additionally, the reason for this phenomenon may be partly attributed to the fact that $0.1 \%$ LC may enhance pig growth by acting as antioxidants. For instance, supplementation of $0.01 \%$ LC in the diet increased the SOD and GSH-Px genes expression in muscle of finishing pigs (Ma et al., 2010). Liu et al. (2008) indicated that dietary LC improves antioxidant ability in ethanol-induced chronic liver injured rats.

The present results showed that ALA administration had no effect on BW gain or carcass traits. This result is consistent with previous reports (Hamano, 2002; Kim et al., 2011). However, there were conflicting results, which showed that there was a reduction in BW in SpragueDawley rats treated with a $0.5 \%$ ALA supplemented highfat diet for 6 weeks (Kim et al., 2011). Shen et al. (2005) reported that dietary $0.5 \%$ ALA significantly decreased weight gain in mice during 3 weeks of the feeding trail compared with mice fed $0 \%$ ALA. They suggested that the BW loss should be related to decreased feed intake as well

Table 4. Effect of dietary L-carnosine (LC) and alpha-lipoic acid (ALA) supplementation on serum lipid profiles in finishing pigs ${ }^{1}$

\begin{tabular}{|c|c|c|c|c|c|c|c|c|}
\hline & \multicolumn{4}{|c|}{ Treatment $^{2}$} & \multirow{3}{*}{ SEM } & \multirow{2}{*}{\multicolumn{3}{|c|}{ Significance (p-value) }} \\
\hline & \multicolumn{2}{|c|}{$\mathrm{LC} 0$} & \multicolumn{2}{|c|}{ LC $0.1 \%$} & & & & \\
\hline & ALA 0 & ALA $0.03 \%$ & ALA 0 & ALA $0.03 \%$ & & $\mathrm{LC}$ & ALA & $\mathrm{LC} \times \mathrm{ALA}^{3}$ \\
\hline $\mathrm{TC}(\mathrm{mmol} / \mathrm{L})$ & 3.39 & 3.20 & 3.09 & 2.99 & 0.214 & $<0.01$ & 0.088 & 0.599 \\
\hline TG (mmol/L) & 0.96 & 0.79 & 0.88 & 0.80 & 0.023 & 0.448 & 0.004 & 0.297 \\
\hline HDL-C (mmol/L) & 0.89 & 0.83 & 1.06 & 0.93 & 0.158 & 0.094 & 0.191 & 0.883 \\
\hline LDL-C (mmol/L) & 3.03 & 2.65 & 3.10 & 2.62 & 0.268 & 0.809 & $<0.01$ & 0.552 \\
\hline VLDL-C (mmol/L) & 0.44 & 0.39 & 0.44 & 0.42 & 0.037 & 0.456 & 0.092 & 0.496 \\
\hline
\end{tabular}

SEM, pooled standard error of the means; TC, total cholesterol; TG, triglyceride; HDL-C, high density lipoprotein cholesterol; LDL-C, low density lipoprotein cholesterol; VLDL-C, very low density lipoprotein cholesterol.

${ }^{1}$ Data were means of 10 pigs from per treatment.

${ }^{2}$ A $2 \times 2$ factorial arrangement with 2 supplemental levels of LC ( 0 or $\left.0.1 \%\right)$ and 2 supplemental levels of ALA ( 0 or $\left.0.03 \%\right)$ in basal diets.

${ }^{3} \mathrm{LC} \times \mathrm{ALA}$, the interaction between LC and ALA. 
as promoted $\beta$-oxidation of fatty acid in mice supplemented with ALA (Kim et al., 2004;2011). The possible reason for these different responses may be that dietary low level ALA has no effect on the growth performance of pigs, while high level ALA supplementing may inhibit the pigs' growth.

The results showed that the groups receiving LC dietary supplementation had significantly reduced serum TC levels compared to those of pigs fed without LC diets, whereas ALA supplementation decreased serum TG and LDL-C levels. Similar to our data, Kim et al. (2011) showed a reduction in serum $\mathrm{TC}, \mathrm{TG}$, and LDL-C levels in rats treated with $0.5 \% \mathrm{LC}$ and/or $0.5 \%$ ALA supplemented highfat diet for 6 weeks. Ford et al. (2001) indicated that ALA supplementation $(300 \mathrm{mg} / \mathrm{kg} \mathrm{BW})$ decreased the plasma TG levels of diabetic rats, and the HDL-C level did not differ. Although the mechanism whereby the LC and ALA exert their effects on serum TC, TG, and LDL-C levels is yet to be investigated (Ford et al., 2001), it may be partly explained by the supplementing of LC and ALA enhancing the activity of lipoprotein lipase (Ichikawa et al., 2005) and increasing the cholesterol metabolism (Marangon et al., 1999) in the liver of pigs. Furthermore, Butler et al. (2009) indicated that the supplementing of ALA in rats diet reduces the level of TG in blood and liver by repressing the gene expression of lipogenic in liver, downregulating liver TG secretion and enhancing the clearance rate of TG.

Kim et al. (2011) reported that combined $0.25 \% \mathrm{LC}$ and $0.25 \%$ ALA supplementation show synergistic effects for increasing skin and liver SOD activities and lipid profiles in rats. However, any synergistic effects by combined $0.1 \%$ LC and $0.03 \%$ ALA supplementation were not found in this study. One possible reason for these different responses may be that the LC or ALA supplemental levels were not sufficient to induce an obvious effect in pigs. The synergistic effect of LC and ALA in pigs is pending investigation.

In conclusion, our results suggest that dietary LC improved ADG in pigs. The growth-promoting effect of LC may be partly related to thyroid hormone changes in pigs. L-carnosine and ALA supplementation decreased serum TC, TG, and LDL-C levels in pigs, which may have the potential to prevent cardiovascular diseases.

\section{ACKNOWLEDGMENTS}

This research was financially supported by the National Program on Key Basic Research Project (973 Program, 2012CB124704), Program for New Century Excellent Talents in University of Ministry of Education of China (2014FG030) and National Key Technology Research and Development Program of the Ministry of Science and Technology of China (2012BAD39B00).

\section{AUTHOR CONTRIBUTIONS}

YH Bao and CQ Gao carried out the experiment trial, performed the statistics and drafted the manuscript. $\mathrm{C}$ Ji and QG Ma conceived and designed the study; WB Hao, T Liu, and LH Zhao carried out the lab analysis; CQ Gao, T Liu, and JY Zhang contributed to data analysis. All authors read and approved the final manuscript.

\section{CONFLICT OF INTEREST}

We certify that there is no conflict of interest with any financial organization regarding the material discussed in the manuscript.

\section{REFERENCES}

Babizhayev, M. A. 2006. Biological activities of the natural imidazole-containing peptidomimetics $n$-acetylcarnosine, carcinine and L-carnosine in ophthalmic and skin care products. Life Sci. 78:2343-2357.

Bai, X. M., Q. G. Ma, L. H. Zhao, L. Xi, and C. Ji. 2012. Effects of alpha-lipoic acid supplementation on antioxidative ability and performance of sows and nursing piglets. J. Anim. Physiol. Anim. Nutr. 96:955-961.

Bellia, F., G. Vecchio, and E. Rizzarelli. 2012. Carnosine derivatives: new multifunctional drug-like molecules. Amino Acids 43:153-163.

Brown, B. E., C. H. J. Kim, F. R. Torpy, C. A. Bursill, L. S. McRobb, A. K. Heather, M. J. Davies, and D. M. van Reyk. 2014. Supplementation with carnosine decreases plasma triglycerides and modulates atherosclerotic plaque composition in diabetic apo E(-/-) mice. Atherosclerosis. 232:403-409.

Bustamante, J., J. K. Lodge, L. Marcocci, H. J. Tritschler, L. Packer, and B. H. Rihn. 1998. Alpha-lipoic acid in liver metabolism and disease. Free Radic. Biol. Med. 24:1023-1039.

Butler, J. A., T. M. Hagen, and R. Moreau. 2009. Lipoic acid improves hypertriglyceridemia by stimulating triacylglycerol clearance and downregulating liver triacylglycerol secretion. Arch. Biochem. Biophys. 485:63-71.

Chen, P., Q. G. Ma, C. Ji, J. Y. Zhang, L. H. Zhao, Y. Zhang, and Y. Z. Jie. 2011. Dietary lipoic acid influences antioxidant capability and oxidative status of broilers. Int. J. Mol. Sci. 12: 8476-8488.

EI-Senousey, H. K., A. M. Fouad, J. H. Yao, Z. G. Zhang, and Q. W. Shen. 2013. Dietary alpha lipoic acid improves body composition, meat quality and decreases collagen content in muscle of broiler chickens. Asian Australas. J. Anim. Sci. 26:394-400.

Ford, I., M. A. Cotter, N. E. Cameron, and M. Greaves. 2001. The effects of treatment with $\alpha$-lipoic acid or evening primrose oil on vascular hemostatic and lipid risk factors, blood flow, and peripheral nerve conduction in the streptozotocin-diabetic rat. Metabolism 50:868-875.

Hamano, Y. 2007. Continuous infusion of lipoic acid rapidly reduces plasma beta-hydroxybutyrate with elevation of non- 
esterified fatty acids in broiler chickens. Br. J. Nutr. 97:495501.

Hamano, Y. 2002. Influence of lipoic acid on lipid metabolism and $\beta$-adrenergic response to intravenous or oral administration of clenbuterol in broiler chickens. Reprod. Nutr. Dev. 42:307-316.

Hamano, Y. 2006. Effects of dietary lipoic acid on plasma lipid, in vivo insulin sensitivity, metabolic response to corticosterone and in vitro lipolysis in broiler chickens. Br. J. Nutr. 95:10941101.

Hamano, Y., S. Sugawara, Y. Kamota, and E. Nagai. 1999. Involvement of lipoic acid in plasma metabolites, hepatic oxygen consumption, and metabolic response to a beta-agonist in broiler chickens. Br. J. Nutr. 82:497-503.

Hamano, Y., Y. Kamota, and S. Sugawara. 2000. Effects of lipoic acid on plasma metabolites and metabolic response to intravenous injection of isoproterenol in broilers. Asian Australas. J. Anim. Sci. 13:653-658.

Ichikawa, T., J. Liang, S. Kitajima, T. Koike, X. Wang, H. Sun, M. Morimoto, H. Shikama, T. Watanabe, N. Yamada, and J. Fan. 2005. Macrophage-derived lipoprotein lipase increases aortic atherosclerosis in cholesterol-fed $\mathrm{Tg}$ rabbits. Atherosclerosis 179:87-95.

Kim, M. S., J. Y. Park, C. Namkoong, P. G. Jang, J. W. Ryu, H. S. Song, J. Y. Yun, I. S. Namgoong, J. Ha, I. S. Park, I. K. Lee, B. Viollet, J. H. Youn, H. K. Lee, and K. U. Lee. 2004. Antiobesity effects of alpha-lipoic acid mediated by suppression of hypothalamic AMP-activated protein kinase. Nat. Med. 10: 727-733.

Kim, M. Y., E. J. Kim, Y. N. Kim, C. Choi, and B. H. Lee. 2011. Effects of $\alpha$-lipoic acid and L-carnosine supplementation on antioxidant activities and lipid profiles in rats. Nutr. Res. Pract. 5:421-428.

Liu, W. H., T. C. Liu, and M. C. Yin. 2008. Beneficial effects of histidine and carnosine on ethanol-induced chronic liver injury. Food Chem. Toxicol. 46:1503-1509.
Ma, X. Y., Z. Y. Jiang, Y. C. Lin, C. T. Zheng, and G. L. Zhou. 2010. Dietary supplementation with carnosine improves antioxidant capacity and meat quality of finishing pigs. J. Anim. Physiol. Anim. Nutr. 94:e286-e295.

Marangon, K., S. Devaraj, O. Tirosh, L. Packer, and I. Jialal. 1999. Comparison of the effect of alpha-lipoic acid and alphatocopherol supplementation on measures of oxidative stress. Free Radic. Biol. Med. 27:1114-1121.

Mong, M. C., C. Y. Chao, and M. C. Yin. 2011. Histidine and carnosine alleviated hepatic steatosis in mice consumed high saturated fat diet. Eur. J. Pharmacol. 653:82-88.

NRC. 1998. Nutrient Requirements of Swine. 10th ed. National Academic Press, Washington, DC, USA.

Qiao, X., H. J. Zhang, S. G. Wu, H. Y. Yue, J. J. Zuo, D. Y. Feng, and G. H. Qi. 2013. Effect of $\beta$-hydroxy- $\beta$-methylbutyrate calcium on growth, blood parameters, and carcass qualities of broiler chickens. Poult. Sci. 92:753-759.

Roy, S. and L. Packer. 1998. Redox regulation of cell functions by $\alpha$-lipoate: Biochemical and molecular aspects. Biofactors 8 : 17-21.

Shay, K. P., R. F. Moreau, E. J. Smith, A. R. Smith, and T. M. Hagen. 2009. Alpha-lipoic acid as a dietary supplement: molecular mechanisms and therapeutic potential. Biochim. Biophys. Acta. General Subjects 1790:1149-1160.

Shen, Q. W., C. S. Jones, N. Kalchayanand, M. J. Zhu, and M. Du. 2005. Effect of dietary alpha-lipoic acid on growth, body composition, muscle $\mathrm{pH}$, and AMP-activated protein kinase phosphorylation in mice. J. Anim. Sci. 83:2611-2617.

Smith, J. W., A. T. Evans, B. Costall, and J. W. Smythe. 2002. Thyroid hormones, brain function and cognition: A brief review. Neurosci. Biobehav. Rev. 26:45-60.

Zhang, Y., K. Hongtrakul, C. Ji, Q. G. Ma, L. T. Liu, and X. X. Hu. 2009. Effects of dietary alpha-lipoic acid on anti-oxidative ability and meat quality in arbor acres broilers. Asian Australas. J. Anim. Sci. 22:1195-1201. 